\title{
ESTUDIO Y ANÁLISIS DE LOS SISTEMAS DE GESTIÓN AMBIENTAL EN EL SECTOR HOTELERO DE QUINTANA ROO
}

\section{STUDY AND ANALYSIS OF AN ENVIRONMENTAL MANAGEMENT SYSTEMS WITHIN THE HOTEL SECTOR OF QUINTANA ROO}

\author{
RAFAel Portillo VAldes \\ Investigador independiente, México \\ rafaporti@hotmail.com
}

\section{RESUMEN:}

El Sistema de Gestión Ambiental, (SGA), como modelo de gestión sobrepasa las declaraciones simbólicas, convirtiéndose en los últimos años en una parte intrínseca de la cultura empresarial que afecta a la empresa, a sus grupos de interés, al medio ambiente y a la comunidad de forma directa. El gran reto que este modelo de gestión radica en saber hallar el balance entre el beneficio obtenido por la empresa, (como generadora de beneficio), y el efecto (positivo o negativo) en su dimensión económica y ambiental. Este sistema de gestión con perspectiva de GA tiene a corto plazo un costo que ha de absorber la empresa, sin embargo, se puede observar que dicho costo pasa a ser una inversión generadora de beneficios tangibles e intangibles en el medio y largo plazo. Dichos beneficios pueden posicionar a la empresa en una posición de clara ventaja competitiva con respecto a su más directa competencia, especialmente en entornos de una competitividad tan agresiva como la que se da en el entorno turístico, donde la oferta existente supera a la demanda, al menos en el segmento de mercado de hoteles cinco estrellas en régimen de todo incluido ubicado en zonas costeras protegidas. Es en estos casos de tan alta agresividad competitiva de mercado donde este SGA puede marcar la diferencia de elección por parte de los usuarios, trabajadores y grupos de interés haciendo a la empresa líder en este sector tan susceptible a dichas dimensiones. 


\section{Palabras clave:}

Sistemas de Gestión Ambiental, estrategia corporativa, medio ambiente, dimensión social, posicionamiento empresarial.

\section{ABSTRACT:}

Environmental Management System (EMS), as a management model goes beyond symbolic declarations, becoming in recent years an intrinsic parto f the business culture which affects the company as such, its stakeholders, the environment and the community directly. The main challenge of this EMS model lies in finding the balance between the benefits obtained by the company (as a generator of profit), and the effect (positive or negative) occurred in its economical and environmental dimension. This management system, with a EMS perspective, has a short term cost which is absorbed by the company, however, such a cost becomes an investment that generates tangible and intangible benefits in the medium and long term. Such benefits can place the company in a clear competitive advantage position with respect of $f$ its more direct competitors, especially in such an aggressive and competitive market as those found in the tourism environment, where existing supply excedes the actual demand, at least in the market segmento ffive-star allinclusive resorts located in protected coastal areas. It occurs in these high competitive market aggressiveness, where the EMS can make the difference when its clients, workers and stakeholders must select who to work with, making the company the leader in this industrial sector so susceptible to such dimensions.

\section{Keywords:}

Environmental Management System, corporate strategy, environment, social dimensión, corporate market positioning.

SUMARIO: I. Introducción. II. Contenido. III. Discusión. IV. Conclusiones. V. Bibliografía.

\section{INTRODUCCIÓN}

Los Sistemas de Gestión Ambiental (SGA) a nivel corporativo son de difícil definición ya que conceptualmente abarcan diferentes dimensiones en diferentes lugares con diferentes personalidades y distintas temporalidades, (Córdoba, 2007). Es por esto que los SGA sobrepasan las declaraciones simbólicas como modelo de gestión teniendo que formularse de una forma ordenada y estructurada que emane desde las mismas estrategias corporativas de la compañía para así contar con el apoyo incondicional, claro y total del consejo de dirección u órgano de gobierno de la misma. De igual importancia es su inclusión de forma permanente en la visión, misión y valores corporativos para así poder comunicarlos de forma efectiva a todos los colaboradores externos e internos, no solo sus políticas de gestión ambiental (GA) sino también los logros obtenidos, el impacto en la sociedad, en el medio ambiente y en sus stakeholders, de hecho hay autores como 
Vonpeltesta, (2014), que van más allá y consideran que lo primero que se debe de tomar en cuenta dentro del plan estratégico de cada empresa es el medio ambiente y a los Stakeholders o partes interesadas y en función de sus demandas y necesidades trabajar hacia el interior para poder satisfacer sus expectativas buscando el beneficio conjunto.

Hay que tener en consideración que los estudios, consensos y normativas sobre la GA han sido ya más que satisfechas desde un marco metodológico teórico en diversos escenarios con parámetros totalmente distintos observando que la implementación del sistema desde la propia creación de la empresa puede ser la clave para que esta sea sustentable en el medio largo plazo. Es por esto que cada vez más empresas y compañías de todos los giros y tamaños se encuentran ante distintos escenarios con distintas alternativas. En algunos casos deciden transformar de forma totalmente voluntaria su estrategia corporativa de negocio para dar cabida a la GA mediante una adecuada gestión del cambio para adaptarse a los nuevos retos del entorno y también contribuir en la medida de sus capacidades al desarrollo de la GA, en otros escenarios la empresa debe actualizar sus políticas de GA a las nuevas condiciones sociales, económicas y ambientales que surgen debido al crecimiento demográfico, el desarrollo tecnológico, la digitalización y el efecto sobre el ecosistema entre otros, también se plantea el escenario donde se ha de implementar un SGA desde cero, no movidos por un ánimo altruista, sino más bien por imperativos legales y en algunos casos por limpieza de imagen tras una catástrofe como fue el caso de la petrolera BP en el Golfo de México.

De este planteamiento surge la motivación de esta investigación que, a su vez, parte de las siguientes preguntas iniciales:

- ¿Es útil el SGA integral para asistir a las empresas (hoteles) en ciernes en la fase de conceptualización y diagnóstico ambiental (DA)?

- ¿Cuáles son las características principales que ha de tener un SGA integral para que resulte efectivo a la hora de minimizar el impacto ambiental (IA) del hotel en el ecosistema?

- ¿Podría este tipo de sistema asociarse con estándares y normas medio ambientales de manera que se logre una integración completa del DA, SGA y mejora continua?

- ¿Cuáles serían las etapas a incorporar para lograr un SGA integral con perspectiva de mejora continua?

- ¿Qué nuevas condiciones sociales, económicas y ambientales que surgen debido al crecimiento demográfico, el desarrollo tecnológico, la digitalización y el efecto sobre el ecosistema se pueden incorporar de manera sencilla y efectiva al SGA integral?

\section{CONTENIDO}

\section{Hipótesis}

Como menciona Hayman (1991), haciendo uso de la hipótesis como el punto de enlace entre la teoría y la observación se tratará de resaltar la relación o 
vínculo entre una o más variables con el fin de elaborar objetivos generales y específicos, seleccionar el tipo de diseño de investigación factible con los retos expuestos así como el método los instrumentos y las técnicas de investigación apropiadas a cada escenario y los recursos humanos y materiales que se emplearán para la consecución en tiempo y forma de la investigación planteada. Tamayo (1989), señala que estas se constituyen en un eslabón imprescindible entre la teoría y la investigación que llevan al descubrimiento de un hecho.

Con relación a las preguntas formuladas en la sección anterior, se sugieren las siguientes hipótesis de partida:

Posibilidad: La generación de ideas dentro de la fase de conceptualización en la etapa de diseño de un SGA puede darse mediante una tormenta de ideas de manera que todos los grupos de interés, tanto internos como externos, se sientan parte de la misma lo que generará un mayor número de ideas con mejor calidad y en mayor profundidad.

Efectividad: La efectividad práctica de un SGA puede ser medida y evaluada mediante la definición de un cuadro de mando integral (CMI). Como explica Kaplan (1996), los CMI pueden ayudar de forma sustancial y ser de gran aporte objetivo a la mejora continua de cualquier proyecto o programa, ende a la empresa.

Estructura: Un SGA orientado a apoyar en todas las fases de un modelo de GA ha de tener al menos 3 elementos fundamentales, un líder plenamente identificado y capacitado técnica y estratégicamente, una dirección de empresa comprometida completamente con el proyecto y un presupuesto propio independiente que evite dependencias de otros departamentos restando importancia que dicho sistema merece.

Método: A partir de evaluaciones de SGA aplicadas a la resolución de retos en sus distintas fases de concepción, diagnosis, implementación y mejora continua, así como en diferentes modelos basados en tamaño de los hoteles, ubicación geográfica, tipo de hotel y nacionalidad, entre otros, es posible identificar elementos relevantes y características particulares, así como técnicas y modelos que debe contener un programa de GA para potenciar y facilitar su implementación integral.

\section{Objetivos}

\section{Objetivo general}

El objetivo principal de este estudio es obtener una estructura que apoye, estratégica y técnicamente la implementación de un SGA integral bajo la normativa adecuada aplicable a cada tipo de hotel en la Riviera Maya sita en el Estado de Quintana Roo, (México), independientemente de su ubicación geográfica, tamaño y nacionalidad, es decir desde una perspectiva macro al Sector Hotelero de la Riviera Maya (SHRM). También como objetivo se plantean posibles estrategias de mejora continua del SGA en aquellos hoteles que ya lo tienen implementado, pero tienen áreas de mejora o se encuentran obsoletos. 


\section{Objetivos específicos}

- Identificación de los hoteles que puedan generar afectaciones al medio ambiente mediante la oferta de sus servicios y/o productos.

- Definición y medida de que las afectaciones causan al medio ambiente, así como posibles soluciones.

- Análisis de los procesos de dichos hoteles que generen afectaciones al medio ambiente.

- Revisión del proceso de implementación del Sistema de Gestión Ambiental en dichos hoteles en caso de existir alguno.

- Plantear opciones y acciones de mejoramiento para los puntos críticos del diseño del SGA.

\section{Metodología}

\section{Definición del tipo de investigación}

En el presente estudio se realiza una investigación interactiva la cual implica la realización de acciones de investigación de forma individual y mediante entrevistas en línea con el fin de obtener la información necesaria para ejecutar un análisis y detectar potenciales retos ambientales de carácter repetitivo en la industria hotelera de la zona de estudio.

La fase interactiva implica la investigación y obtención de datos con el fin de poder identificar y describir retos repetitivos y relevantes que de alguna manera entorpezcan la aplicación de un SGA integral, así como la introducción de mejoras continuas en el proceso.

Fases de la investigación:

Exploratoria: Integra el proceso de exploración, minería y revisión de estudios existentes de corte descriptivo, comparativo, analítico y explicativo entre otros, a la vez que se plantea un enunciado interactivo mientras se detecta un evento a modificar.

Descriptiva: Se describen los principales retos encontrados que justifican una acción propuesta mientras que se identifican las necesidades de cambio, planteando objetivos generales.

Comparativa: Se revisan las investigaciones realizadas con los retos a modificar y/o crear y sus posibles procesos causales con una comparación de conceptos y teorías.

Analítica: Análisis sintagmático de las teorías sobre los retos hallados y necesitados de cambios, así como de los procesos causales.

Proyectiva: En esta fase se da una descripción o diagnóstico buscando posibles explicaciones a la causalidad de los retos existentes, así como la elaboración de predicciones de hipótesis o escenarios futuros con el fin de elaborar un plan de acción ejecutable y perdurable en el tiempo basado en la mejora continua. 


\section{Diseño de la investigación}

Evento del estudio: Un SGA integral en el sector hotelero de la Riviera Maya (SHRM), sita en el Estado de Quintana Roo, (México). Las Normas internacionales sobre Gestión Ambiental tienen como finalidad proporcionar a las organizaciones los elementos de un sistema de gestión ambiental (SGA) eficaz que puedan ser integrados con otros requisitos de Gestión, y para ayudar a las organizaciones a lograr metas ambientales y económicas dentro de la legislación medio ambiental vigente.

Unidad de estudio: Sector hotelero de la Riviera Maya (SHRM), en el Estado de Quintana Roo, (México), con especial incidencia en aquellos hoteles que incumplen de forma voluntaria o involuntaria con la legislación ambiental vigente, coludidos o no con el gobierno y funcionarios en turno.

\section{Descripción de los elementos metodológicos utilizados}

Para el análisis de los procesos del SHRM que pueden generar afectaciones al medio ambiente se usan diferentes técnicas tales como:

Análisis de datos estadísticos de la afectación a zonas y especies protegidas como los humedales a nivel república, estatal y municipal a través del Instituto Nacional de Estadística y Geografía (INEGUI) basados principalmente en el efecto producido por el SHRM en el Estado de Quintana Roo. Se emplean datos históricos para analizar la afectación negativa del SHRM en el medio ambiente y poder identificar hechos causales para conocer actuaciones concretas mediante análisis de algunos procesos de hoteles específicos que se usan como ejemplo muestra.

Se extiende dicha investigación y análisis a un número de hoteles que sirva de muestra real correlativa con el fin de saber en qué estado se hayan sus SGA integral en caso de tener uno y en caso de ausencia del mismo determinar las razones.

Esto conlleva al planteamiento de opciones y acciones de mejoramiento para los puntos críticos del diseño del SGA y que son comunes a la mayor parte del SHRM. 


\section{Diseño metodológico}

Figura 1: Diseño metodológico

\begin{tabular}{|c|c|c|c|c|}
\hline RESULTADO & ACTIVIDADES & TÉCNICAS & INSTRUMENTOS & PRODUCTO \\
\hline \multirow{3}{*}{$\begin{array}{l}\text { Análisis de los } \\
\text { procesos del } \\
\text { SHRM que } \\
\text { pueden generar } \\
\text { afectaciones al } \\
\text { medio ambiente }\end{array}$} & $\begin{array}{l}\text { Identificación y } \\
\text { análisis de los } \\
\text { procesos del } \\
\text { SHRM }\end{array}$ & $\begin{array}{c}\text { Análisis } \\
\text { Organizacional }\end{array}$ & $\begin{array}{l}\text { Mapa de } \\
\text { procesos }\end{array}$ & \multirow{3}{*}{$\begin{array}{l}\text { Informe de } \\
\text { estado } \\
\text { ambiental del } \\
\text { SHRM }\end{array}$} \\
\hline & $\begin{array}{c}\text { Reconocimiento } \\
\text { de los aspectos } \\
\text { ambientales del } \\
\text { SHRM }\end{array}$ & $\begin{array}{l}\text { Investigación y } \\
\text { análisis de data } \\
\text { pública oficial }\end{array}$ & $\begin{array}{l}\text { Gráficas } \\
\text { producidas }\end{array}$ & \\
\hline & $\begin{array}{l}\text { Identificación del } \\
\text { nivel de } \\
\text { afectación de los } \\
\text { aspectos e } \\
\text { impactos } \\
\text { ambientales del } \\
\text { SHRM }\end{array}$ & Semaforización & $\begin{array}{l}\text { Matriz de } \\
\text { valorización en } \\
\text { forma de gráfica }\end{array}$ & \\
\hline $\begin{array}{l}\text { Revisión del } \\
\text { proceso de } \\
\text { implementación } \\
\text { del SGA en el } \\
\text { SHRM }\end{array}$ & $\begin{array}{c}\text { Análisis de las } \\
\text { necesidades y } \\
\text { retos } \\
\text { ambientales del } \\
\text { SHRM conforme } \\
\text { a normativa legal } \\
\text { medio ambiental } \\
\text { vigente }\end{array}$ & $\begin{array}{l}\text { Revisión } \\
\text { documental, } \\
\text { Revisión de } \\
\text { campo }\end{array}$ & $\begin{array}{c}\text { Bases } \\
\text { documentales }\end{array}$ & $\begin{array}{l}\text { Estado actual del } \\
\text { SGA en el SHRM }\end{array}$ \\
\hline \multirow{2}{*}{$\begin{array}{l}\text { Planteamiento } \\
\text { de opciones y } \\
\text { acciones de } \\
\text { mejoramiento } \\
\text { para el SGA en el } \\
\text { SHRM }\end{array}$} & $\begin{array}{c}\text { Análisis de los } \\
\text { resultaos } \\
\text { obtenidos }\end{array}$ & \multirow{2}{*}{$\begin{array}{c}\text { Revisión } \\
\text { documental, } \\
\text { Sondeo, Análisis } \\
\text { de contenido. }\end{array}$} & \multirow{2}{*}{$\begin{array}{l}\text { Matriz de } \\
\text { revisión y } \\
\text { criterios de } \\
\text { mejora DOFA }\end{array}$} & \multirow{2}{*}{$\begin{array}{c}\text { Opciones de } \\
\text { mejora del SGA } \\
\text { en el SHRM }\end{array}$} \\
\hline & $\begin{array}{l}\text { Integración de } \\
\text { propuestas }\end{array}$ & & & \\
\hline
\end{tabular}

Fuente: Elaboración propia.

Resultados

\section{Análisis del macro y micro entorno ambiental}

Como primer paso se realiza una revisión de la situación medio ambiental de una perspectiva macro reduciéndola al área de interés del presente estudio. Se puede observar que a nivel ambiental el principal objetivo es mostrar la importancia de los SGA dentro del marco legislativo de la GA. Esto se hace tomando como referencia la desforestación que han sufrido los manglares a nivel mundial para ir centrándose en México, la Península del Yucatán (compuesta por los estados de Campeche, Yucatán y Quintana Roo), el estado de Quintana Roo y la Riviera Maya dentro de este. 
Se estima que la superficie de manglares a nivel mundial es de 15.2 millones de hectáreas; las zonas más extensas se encuentran en Asia y África, seguidas por América del Norte y Central. Desde 1980 se ha perdido la nada despreciable superficie de un $20 \%$, o 3.6 millones de hectáreas.

En los últimos lustros, la tasa de pérdida neta parece haber disminuido, pero no a un ritmo que permita recuperar el manglar perdido. En la década de 1980 alrededor de 185,000 hectáreas se perdieron por año; esta cifra cayó a 118,500 hectáreas por año en la década de 1990 y a 102,000 hectáreas $(-0.66 \%)$ en el período 2000-2005, lo cual refleja que ha aumentado el conocimiento sobre el valor de los ecosistemas de manglares (aunque siempre con sus excepciones regionales o locales que no consideran el daño ecológico producido) (ELAW, 2007).

Figura 2: Superficie hectáreas manglar en el mundo 1980-2005

\begin{tabular}{|c|c|}
\hline AÑO & superficie hectáreas manglar planeta \\
\hline 1980 & 15200000 \\
\hline 1985 & 15107500 \\
\hline 1990 & 15015000 \\
\hline 1995 & 14422500 \\
\hline 2000 & 13830200 \\
\hline 2005 & 13320200 \\
\hline
\end{tabular}

\section{superficie hectáreas manglar planeta}

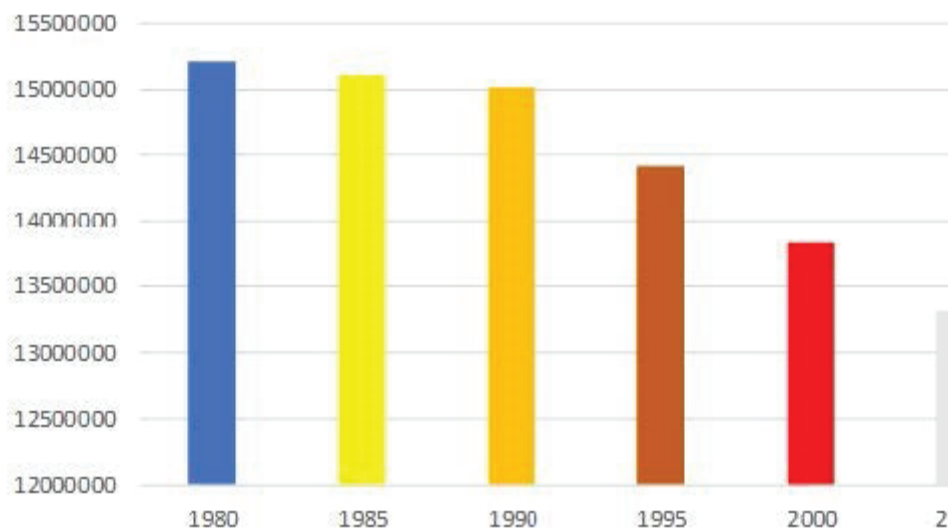

Fuente: Elaboración propia a partir Sistema de Monitoreo de Manglares de México. 
En la figura 2 se puede observar gráficamente como ha ido desarrollándose la conciencia medioambiental a través de una apropiada macro GA ende los indicadores de sustentabilidad a nivel mundial permiten ahora tener una visión del costo real no tener un SGA apropiado, no solo a nivel económico sino también teniendo en cuenta las dimensiones ecológica y social.

Reduciendo el espectro de superficie de hectáreas de manglar a nivel México se detallan las siguientes cifras observables en la figura 3, donde con un patrón de inicio similar se puede observar una ligera recuperación del mangle debido a una legislación medio ambiental más estricta a nivel mundial y nacional promovida por supra políticas provenientes de organismos como la ONU con el beneplácito de sus países miembros en la mayoría de los casos que asumen los objetivos a medio y largo plazo acordados en dichas instancias supranacionales.

Figura 3: Superficie hectáreas manglar en México 1981-2015

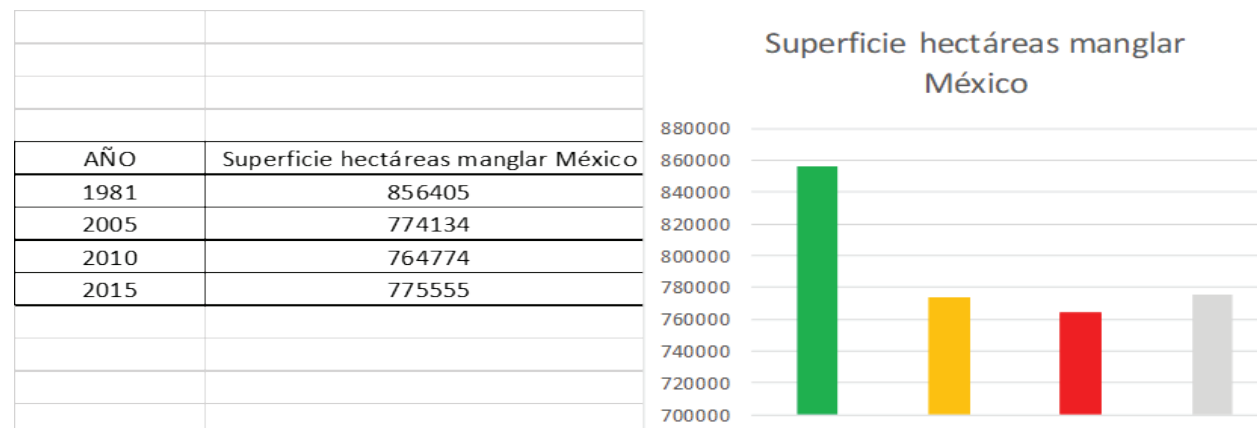

Fuente: Elaboración propia a partir Sistema de Monitoreo de Manglares de México.

En la figura 3, reduciendo el espectro geográfico al país de México se puede observar una disminución similar en proporción al número de hectáreas en el planeta con la diferencia que México ha tenido un claro repunte a partir del año 2005 en la repoblación de zonas humedales de manglar debido a los planes de reforestación de los mismos por parte del gobierno federal. En México una importante superficie de manglar ha sido afectada de forma indirecta por obras de infraestructura que han alterado los flujos hidrológicos, como presas, bordos, canales y dragados, a lo cual se suman fenómenos naturales como los huracanes que contribuyen con su destrucción principalmente en las zonas costeras del Caribe y Océano Pacífico, así como las construcciones y desarrollos de zonas turísticas en áreas costeras como es el caso, entre otros, del Estado de Quintana Roo en la Península de Yucatán.

Si se reduce el marco geográfico del estudio a la península de Yucatán con sus tres estados, Campeche, Yucatán y Quintana Roo se puede observar un movimiento uniforme en cuanto a la degradación del manglar, así como su reforestación en sus tres estados (figura 4), no siendo dicha reforestación lo suficientemente 
amplia como para regenerar el manglar arrasado a una velocidad mayor o igual que su desforestación, siendo este un claro ejemplo del indicador de la Huella Ecológica como menciona Wackernagel (2001). De hecho, en algunos casos la reforestación se antoja imposible debido al daño causado por construcciones diversas sobre el propio humedal o desertización del mismo.

Figura 4: Hectáreas de manglar en la Península de Yucatán por estado

\begin{tabular}{|c|c|c|}
\hline AÑO & ESTADO & Superficie Hectáreas Manglar por Estado \\
\hline 1980 & Yucatán & 98756 \\
\hline 2005 & Yucatán & 91701 \\
\hline 2010 & Yucatán & 91348 \\
\hline 2015 & Yucatán & 93171 \\
\hline 1980 & Quintana Roo & 137910 \\
\hline 2005 & Quintana Roo & 130210 \\
\hline 2010 & Quintana Roo & 128048 \\
\hline 2015 & Quintana Roo & 129902 \\
\hline 1980 & Campeche & 216969 \\
\hline 2005 & Campeche & 199662 \\
\hline 2010 & Campeche & 197623 \\
\hline 2015 & Campeche & 198853 \\
\hline
\end{tabular}

\section{Superficie Hectáreas Manglar por Estado}
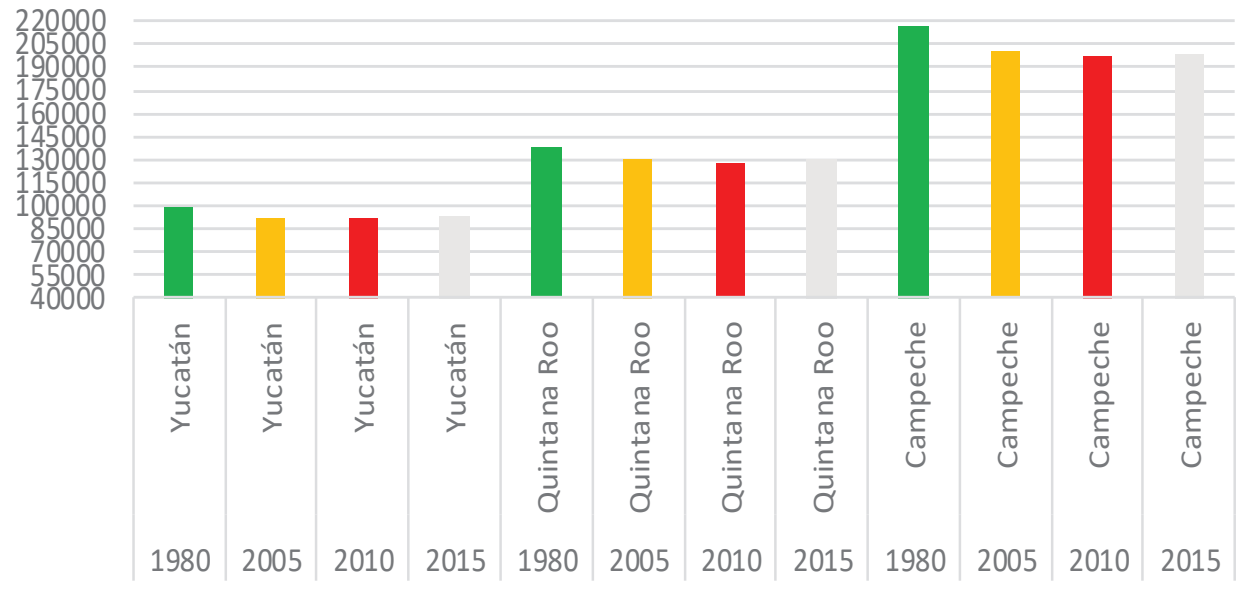

Fuente: Elaboración propia a partir Sistema de Monitoreo de Manglares de México. 
En la figura 4 como se menciona anteriormente se puede observar la Huella Ecológica dejada por el turismo y su desmesurada construcción de zonas turísticas sin preocuparse por la GA. Sin embargo, al comenzar a introducirse los impuestos ecológicos y tener en cuenta los ajustes económicos por deterioro y uso del medio ambiente, así como los gastos en protección ambiental (figura 5) y demás medidas protectoras del medio ambiente mediante SGA integral, dicha desforestación ha decrecido gracias a la inversión de esta nueva recaudación y la posibilidad de ver el costo real de las inversiones teniendo en cuenta dicho costo ecológico. A nivel macroeconómico este tipo de tasa o impuestos se pueden ver de la siguiente manera: 
Figura 5: Indicadores tradicionales vs. Indicadores de GA

\begin{tabular}{|c|c|c|c|c|c|}
\hline Año & $\begin{array}{l}\text { Producto Interno } \\
\text { Bruto (PIB ) }\end{array}$ & $\begin{array}{l}\text { Producto Interno } \\
\text { Neto Ajustado } \\
\text { Ambientalmente } \\
\text { (PINE) }\end{array}$ & $\begin{array}{l}\text { Costos Totales } \\
\text { por Agotamiento } \\
\text { y Degradación } \\
\text { (CTADA) }\end{array}$ & $\begin{array}{c}\text { Gastos en } \\
\text { Protección } \\
\text { Ambiental (GPA) }\end{array}$ & PINE/PIB \\
\hline 2003 & $7,868,810$ & $6,185,997$ & 534,713 & 40,010 & 78.6 \\
\hline 2004 & $8,828,367$ & $7,010,697$ & 532,554 & 43,620 & 79.4 \\
\hline 2005 & $9,562,648$ & $7,600,515$ & 583,770 & 52,973 & 79.5 \\
\hline 2006 & $10,630,939$ & $8,486,006$ & 626,448 & 61,383 & 79.8 \\
\hline 2007 & $11,504,076$ & $9,226,511$ & 625,036 & 76,018 & 80.2 \\
\hline 2010 & $13,366,377$ & $10,481,157$ & 772,072 & 108,000 & 78.4 \\
\hline 2011 & $14,665,576$ & $11,575,351$ & 794,277 & 129,631 & 78.9 \\
\hline 2012 & $15,817,755$ & $12,431,465$ & 877,429 & 126,029 & 78.6 \\
\hline 2013 & $16,277,187$ & $12,807,727$ & 902,230 & 117,228 & 78.7 \\
\hline 2014 & $17,473,842$ & $13,893,100$ & 861,659 & 121,905 & 79.5 \\
\hline 2015 & $18,551,459$ & $14,645,556$ & 866,974 & 116,348 & 78.9 \\
\hline
\end{tabular}

Indicadores tradicionales vs. indicadores de sustentabilidad

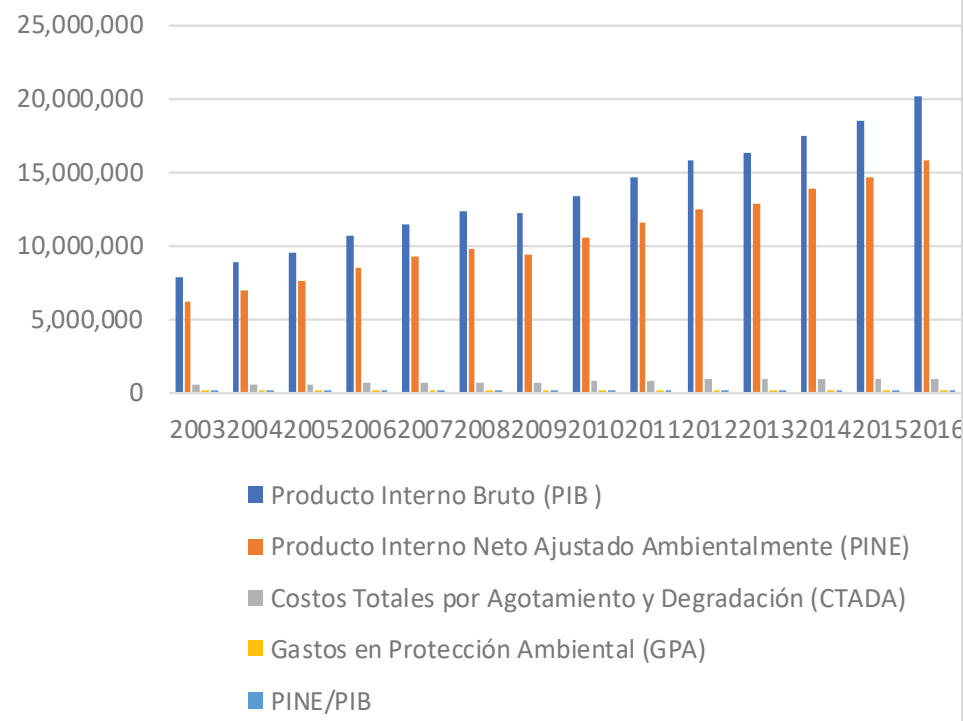

Fuente: Elaboración propia a partir de datos de inegi.org.mx 
Los indicadores de GA tienen una base empírica basada en distintos desarrollos realizados en los últimos lustros por sus principales impulsores como Wen Jiabao o el economista Serge Latouche. Una observación del PNB al PNBe quedaría de la siguiente manera:

Modificación del PNB al PNBe

$\mathrm{PNBE}=\mathrm{PNB}+\mathrm{SA} \pm \mathrm{DA} 1-\mathrm{GD}-\mathrm{CU}$

PNBe: Producto Nacional Bruto Ecológico

SA: Servicios ambientales. Propuesta de Peskin (1989)

- DA: Daños ambientales. Propuesta de Peskin (1989)

+ DA1: Daños ambientales. Propuesta de Harrison (1989)

GD: Gastos defensivos. Propuesta de Leipert (1986), Juster (1973)

CU: Coste del usuario. Propuesta de El Serafy (1989)

Fuente: Elaboración de Durán, (2005) a partir de Hamilton, (1994).

De la misma manera se pueden calcular otros indicadores tradicionales (PIB, PNN, IPC, etc.) desde un punto de vista ecológico, es decir, añadiéndoles sus respectivas cargas en el uso de los recursos naturales y efecto en el medio ambiente causados en su producción y en algunos casos en su consumo (reciclamiento de residuos sólidos, orgánicos, etc.).

Tomando a la Península de Yucatán como un todo, también se observa en la figura 6 la abrumadora desforestación en toda la península (la cual ni ha podido ser recuperada ni lo será nunca en su totalidad) en la década de los 80-90 hasta 2005 donde empiezan a hacer efecto las políticas implementadas por las instituciones y secretarias creadas durante la época y comienza una reforestación en términos relativos con los datos del 2010 y hasta el 2015. 
Figura 6: Hectáreas totales de manglar en la Península de Yucatán

\begin{tabular}{|l|c|c|}
\hline & Año & $\begin{array}{c}\text { Superficie hectáreas manglar } \\
\text { Peninsula Yucatán }\end{array}$ \\
\hline & 1980 & Hectáreas \\
\hline Península Yucatán & 2005 & 453635 \\
\hline Península Yucatán & 2010 & 421573 \\
\hline Península Yucatán & 2015 & 417019 \\
\hline Península Yucatán & & 421926 \\
\hline
\end{tabular}

\section{Superficie hectáreas manglar Peninsula Yucatán Hectáreas}

\section{0 \\ 440000 \\ 420000 \\ 400000 \\ 380000}
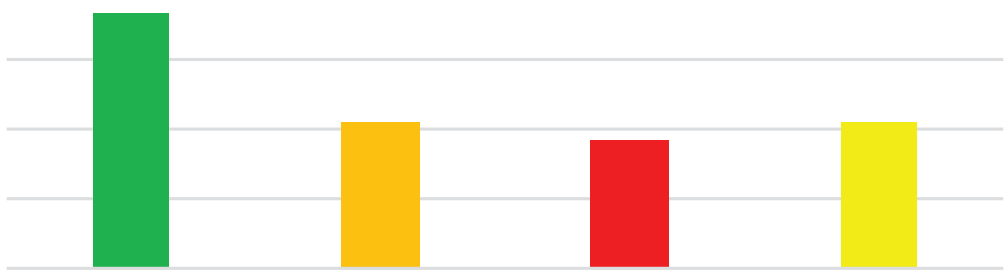

1980

2005

2010

2015

Fuente: Elaboración propia a partir Sistema de Monitoreo de Manglares de México.

Siguiendo con el enfoque micro geográfico se puede observar en la figura 7, que el estado de Quintana Roo, siendo el estado que más turistas atrae del país con una cifra de 26,414,399 de viajeros en el año 2019 (Secretaría de Turismo del Estado de Quintana Roo) es en proporción, el que menos daño ha causado de forma global al entorno de los humedales ya que, si se extrapola esa cantidad de viajeros a los otros dos estados vecinos la desforestación del manglar habría sido notablemente más alta. 
Figura 7: Hectáreas totales de manglar en Quintana Roo

\begin{tabular}{|c|c|c|}
\hline AÑO & ESTADO & Superficie Hectáreas Manglar \\
\hline 1980 & Quintana Roo & 137910 \\
\hline 2005 & Quintana Roo & 130210 \\
\hline 2010 & Quintana Roo & 128048 \\
\hline 2015 & Quintana Roo & 129902 \\
\hline
\end{tabular}

\section{Superficie Hectáreas Manglar}

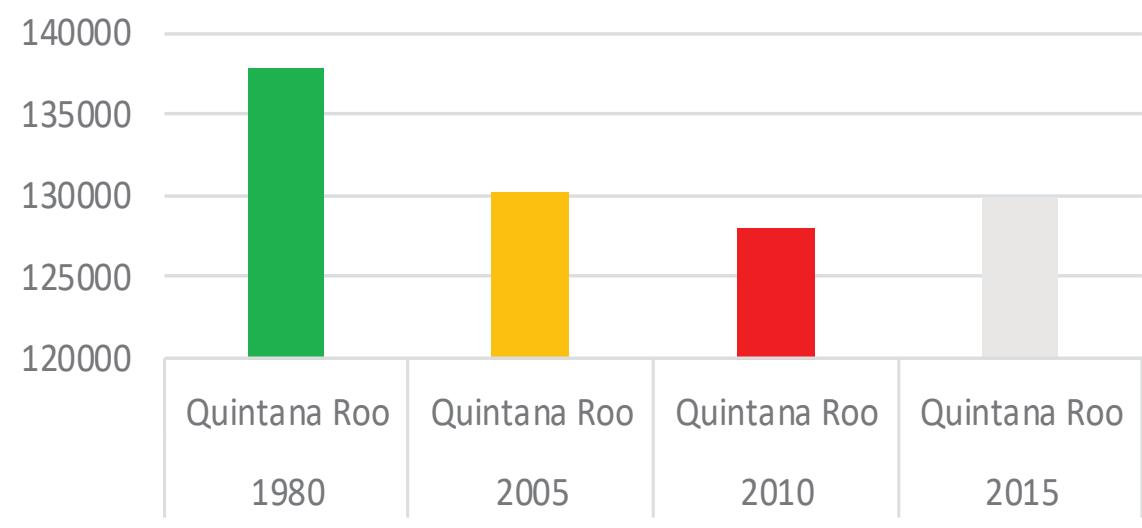

Fuente: Elaboración propia a partir Sistema de Monitoreo de Manglares de México.

\section{Indicadores de SGA vs. indicadores tradicionales}

La importancia de los indicadores SGA integrales Vs sistemas tradicionales radica en que los últimos no toman en cuenta la perspectiva completa del medio ambiente, sus costos asociados, (como puede observarse en la figura 5 y posterior fórmula), y el deterioro en la calidad de vida desde una perspectiva ambiental. Estos hechos pueden verse reflejados en la figura 8 la cual refleja la diferencia entre el uso de indicadores de SGA y los indicadores tradicionales, arrojando estos últimos unos datos irreales e inconsistentes con la realidad de los costos desde un punto de vista de la GA donde se tienen en cuenta los costos intangibles (que ya se pueden cuantificar) ocasionados al medioambiente y que repercuten directamente no solo en la futura continuidad de la empresa (hotel en este caso) sino también en sus colaboradores internos, externos y en el ecosistema en general. 


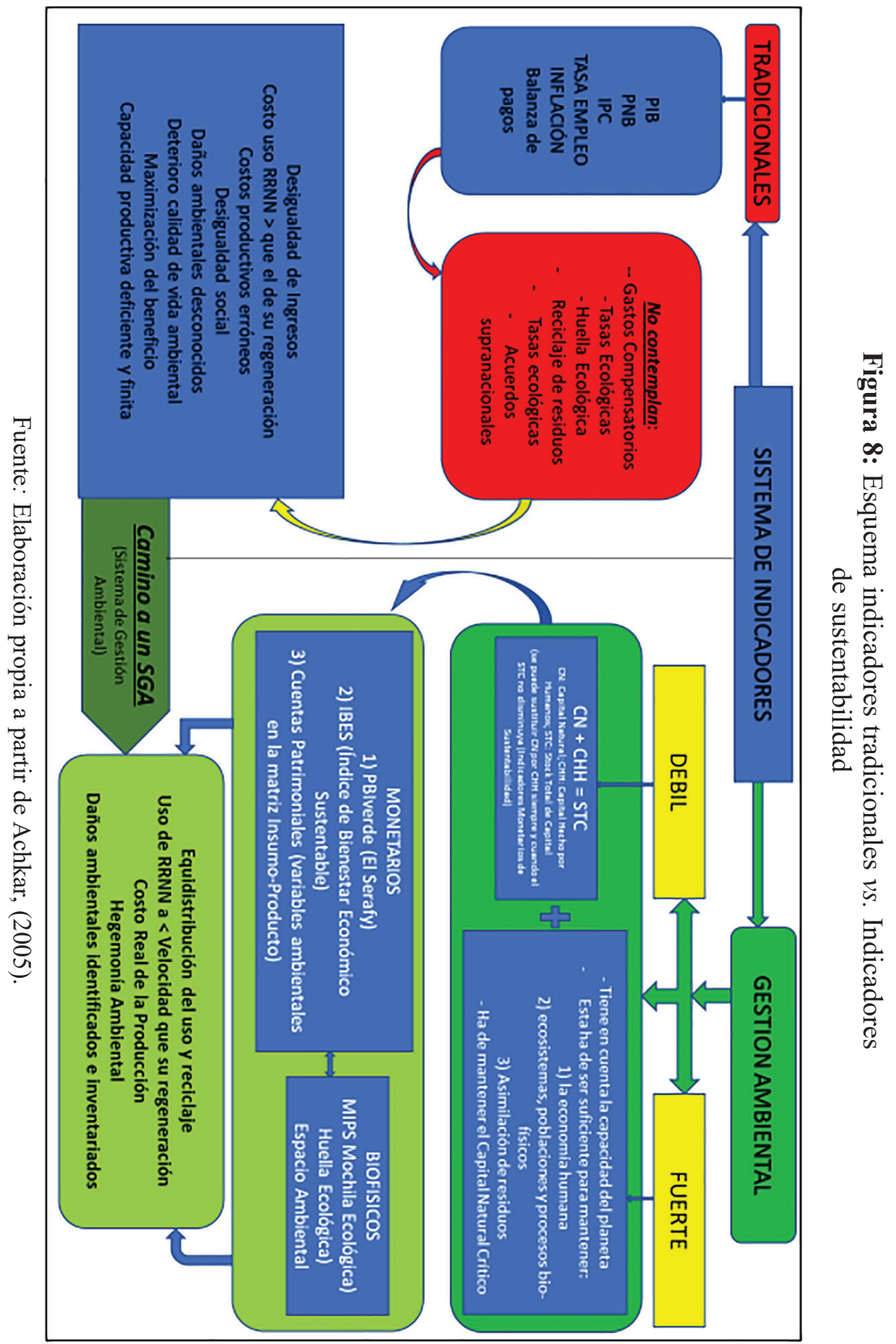


Cabe mencionar que, de forma objetiva, los indicadores de SGA son, aunque sumamente complejos en su elaboración debido a la gran cantidad de variables tangibles e intangibles existentes, mucho más certeros en cuanto a sus mediciones desde las distintas dimensiones medio ambientales que forma la GA y por tanto, totalmente necesarios a la hora de elaborar cálculos, figurar posibles sanciones, hacer estudios y futuras predicciones sobre cuánto, cómo y cuándo se puede consumir con relación al medio ambiente por el SHRM y a qué velocidad, sin dañar el capital ofrecido por el planeta, tan solo usando lo proveniente de sus "intereses" ambientales.

\section{Revisión del proceso de implementación del SGA del SHRM}

Para examinar el estado del SGA en el SHRM se revisó el desarrollo de los requisitos de la Norma ISO 14001:2004, de esta forma se podrá evaluar el avance del SGA en todo su contexto, tanto el estado ambiental como el estado administrativo desde un punto de vista macro empresarial a nivel del SHRM, así como microempresarial a nivel de hoteles individuales.

El propósito de este programa es proporcionar el conocimiento mínimo para comprender los problemas ambientales que genera el SHRM en general y sus componentes en particular, las oportunidades que existen para que los procesos de construcción, generación y distribución de servicios y mantenimiento en general de los mismos, sean menos impactantes y las oportunidades para desarrollar la mejora continua en la resolución y prevención de los retos presentes y futuros.

\section{Planteamiento de opciones y acciones de mejoramiento para el SGA del SHRM}

Desde una perspectiva macro del SHRM, la norma específica en este punto que se debe definir y documentar en el alcance del SGA del SHRM es aún muy difusa por intereses económicos varios, sin embargo, esta macro definición ha de ser extraída de la legislación vigente siendo este uno de los puntos a proponer en el presente documento ya que esta servirá de punto de partida para subsecuentes SGA a nivel hotel.

Con referencia a los hoteles como entes empresariales individuales, en muchos casos el alcance del SGA se definió mas no se documentó, lo cual es necesario realizarlo para que se tenga como evidencia y se haga un buen control en la documentación del SGA para una posterior auditoria. Es por esto que antes de dar este primer paso se recomienda una auditoría de GA a todos os hoteles sitos en la Riviera Maya y que pueden de forma clara y sin lugar a dudas afectar de forma negativa al medio ambiente en la provisión de sus servicios y/o productos.

Dicha auditoría se basará en los requisitos legales en la legislación ambiental vigente del Estado (normalmente decretos y resoluciones) lo cual dará paso a obligaciones específicas para enmarcarse dentro de la legislación actual en la provisión de sus servicios y/o productos que afectan a su actividad relacionada. 


\section{DISCUSIÓN}

Un SGA proporciona un modelo estructurado para la consecución de mejoras continuas con un ritmo de aplicación y extensión que debe ser determinado por el alcance del sector a aplicar o las empresas que pudieran conformarlo. En este caso específico desde una macroperspectiva, lo forma el SHRM compuesto a su vez por los hoteles de distintos tamaños, nacionalidades, ubicaciones geográficas y mesas directivas.

El sistema por sí mismo no produce una reducción de los impactos medioambientales negativos (aparte de los derivados de la disciplina y del control sistemático de los procesos), sin embargo, si capacita el SHRM para alcanzar y controlar sistemáticamente el nivel de comportamiento medioambiental que se proponga en su sector.

Por tanto, este sistema facilitará al SHRM para:

- Identificar los aspectos medioambientales asociados a sus actividades para determinar los impactos ambientales significativos.

- Establecer una política medioambiental adecuada para la organización mediante la identificación el marco legal correspondiente.

- Identificar las prioridades y fijar los objetivos y metas medioambientales.

- Establecer un plan para llevar a cabo la política medioambiental y alcanzar los objetivos.

- Ser capaz de adaptarse a circunstancias cambiantes.

El haber cubierto un primer ciclo de los requisitos de la ISO 14001 y la ISO 37001:2016 no es sinónimo de que su comportamiento medioambiental y éticomoral haya alcanzado su cota más alta, ni que haya eliminado de forma inmediata todos sus impactos medioambientales negativos, por lo que se hace necesario repetir el ciclo en forma periódica y en cada uno de ellos hacer los ajustes correspondientes al sistema. De esta manera se cumplirá con el objetivo de la norma: la mejora continua.

En consecuencia, no basta con obtener la certificación, hay que continuar con el proceso de mejora. Por este motivo los hoteles certificados del SHRM deben someterse a auditorías periódicas de seguimiento y revisión de la certificación. Si al final de cada ciclo del SGMA propuesto (a partir del segundo), los informes de auditoría medioambiental son favorables con respecto al cumplimiento de la política ambiental y al logro de objetivos y metas, entonces se estaría dando la mejora continua, por consiguiente, se podría constatar que el SHRM habrá mejorado su comportamiento medioambiental y ético-moral, incidiendo consecuentemente en la mejora de la calidad de vida que se ofrece a la comunidad Quintanarroense.

\section{CONCLUSIONES}

A modo de conclusión se puede mencionar que se observa una gran área de mejora en lo relativo al aspecto ambiental y ético-moral con una falta de ejemplo 
flagrante por parte de la dirección corporativa del SHRM y sus hoteles en lo que al medio ambiente y manejo de capitales se refiere.

Se considera que habría que hacer un estudio en profundidad desde una perspectiva de GA y ética-moral con el fin de poder medir y analizar los efectos nocivos de este sector a gran escala y escala micro, así como códigos de ética y conducta del SHRM, así como su cumplimiento o no, para poder discernir el impacto (en este caso negativo) que puede tener sobre el ecosistema y la sociedad en sí.

Sería mediante este estudio que se pudiera, si el análisis lo requiere, considerar el obtener una certificación como la ISO 14001 y la ISO 37001:2016 que versan sobre SGA y anticorrupción y ética empresarial respectivamente, pudiendo de esta manera desvincularse de las acciones del SHRM algunos de los hoteles miembros que tanto daño pueden sufrir debido a la mala praxis de algunas cadenas hoteleras del sector.

\section{Retos y obstáculos identificados}

Los principales retos y obstáculos identificados se basan no solo en la perspectiva de la GA como tal sino también en la dimensión ético-moral y algunas de las acciones supuestamente llevadas a cabo por miembros de las juntas directivas del SHRM en detrimento del ecosistema de la Riviera Maya.

Es por esto que si se toman los retos (debilidades) como situaciones endógenas del SHRM, entonces el propio sector hotelero y sus componentes, tienen la capacidad propia de convertir por sus propios medios dicha debilidad en una fortaleza. El cómo hacerlo dependerá del grado de compromiso ético-moral de los dueños/directivos a la hora de manifestarse (con hechos y palabras), de forma clara, rotunda y pública su postura ante este tipo de acciones perniciosas para la economía local y el medio ambiente.

Desde el punto de vista de obstáculos (amenazas) identificadas, como situaciones exógenas al SHRM y a sus componentes, se considera que pueden convertirlas en oportunidades mediante la elaboración y revisión pública, ante sus grupos de interés, de sus códigos y normas de conducta siendo además auditados y evaluados por una empresa externa con el fin de conseguir la certificación ISO 37001:2016, como prueba fehaciente de su compromiso para con el aspecto éticomoral que es el que actualmente está dañando de forma severa la imagen y credibilidad del SHRM y sus componentes.

\section{Probabilidades de éxito}

Se considera que el SHRM tiene escasas posibilidades de éxito en cuanto a la implementación de las normativas ISO 14001 y ISO 37001:2016 respectivamente dado que estas son otorgadas por una empresa externa sin vinculación al SHRM ni a los hoteles que lo conforman y dadas las supuestas recientes acusaciones abiertas por motivos tales como blanqueo de capitales, evasión de 
impuestos, fraude y sobornos (Cruz, 2019), entre otras, se antoja harto difícil el obtenerla en un futuro cercano.

Con respecto a la dimensión ambiental, dada la constante y agresiva competencia existente en la Riviera Maya (México), hay pocas posibilidades que el SHRM pueda continuar con un crecimiento basado en los SGA sino es a cambio de la invasión de los humedales protegidos con la construcción de sus Resorts. Con este tipo de invasiones se considera muy baja la probabilidad de éxito a la hora de tratar de obtener la certificación ISO 14001en Sistemas de Gestión Ambiental.

\section{Recomendaciones y aprendizaje obtenido}

Las principales recomendaciones son la elaboración, aplicación y comunicación de un código de ética y conducta dentro de las empresas que conforman el SHRM que sea realista, honesto y pueda ser puesto en práctica a pesar de las actitudes de algunos individuos y de la tremenda competencia existente en este segmento de mercado en la Riviera Maya.

Una vez elaborados, implementados y transmitidos dichos SGA junto con códigos ético-morales y conductuales se puede proceder a obtener las certificaciones externas internacionales al respecto con el fin de dar la credibilidad y compromiso necesario del SHRM en los aspectos de GA y ético-morales.

En lo referente al aprendizaje obtenido en este ámbito empresarial, es observa que una competencia tan agresiva en ecosistemas protegidos, hace muy difícil el poder mantener unos estándares básicos de educación medio ambiental y SGA, y cuyos devastadores efectos, en algunos casos, tratan de ocultarse mediante aportaciones a la sociedad y al medio ambiente en una cuantía claramente insuficiente al daño causado; Es por esto que se recomienda que todas las partes involucradas en cualquier acción que dañe al medio ambiente de forma continuada e irreparable sean obligadas a pagar sanciones de cuantía económica tres veces mayor al beneficio obtenido por dichas acciones y en su caso, al derribo de las construcciones realizadas sobre zonas protegidas y posterior reforestación en la medida de lo posible.

En lo referente a las actuaciones de individuos en temas de sobornos, evasión de impuestos, etc., se considera que es un tema que ha de cambiar con las nuevas generaciones a través de una adecuada educación donde se enseñe de forma natural que el valor de las acciones correctas es mayor al beneficio tangible obtenido por las incorrectas y que forme parte intrínseca y natural de todos los SGA.

\section{BIBLIOGRAFÍA}

ACHKAR, Marcel, 2005. Indicadores de sustentabilidad. Laboratorio de Desarrollo Sustentable y Gestión Ambiental del Territorio. Departamento de Geografía, Uruguay, Facultad de Ciencias.

Biodiversidad mexicana, 2020. Manglares: ¿Qué son los manglares?, México, CONABIO. Recuperado de https://www.biodiversidad.gob.mx/ecosistemas/ manglares 
CHAPMAN, Alan, 2004. Análisis DOFA y análisis PEST. Recuperado de https:// degerencia.com/articulo/analisis_dofa_y_analisis_pest/

Comisión Nacional de Áreas Naturales Protegidas, 2017, Manglares de Nichupté, joya de biodiversidad. México, CONANP. Recuperado de https://www.gob. $\mathrm{mx} /$ conanp/articulos/manglares-de-nichupte-joya-de-biodiversidad?idiom=es

CÓRDOBA, José Rodrigo y CAMPBELL, Thomas 2007. Iniciativas de implementación de la RSC - Contribución del pensamiento sistémico. Revista científica Pensamiento y Gestión. No 23. pp. 112-130.

CRUZ, Laura, 2019. Destapa corrupción pleito de los Chapur. Luces del Siglo. Cancún, México. Recuperado de https://lucesdelsiglo.com/2019/12/30/anuario2019-destapa-corrupcion-pleito-de-los-chapur/

ELAW Environmental Law Alliance Worlwide, 2007. Los Manglares del Mundo (48). Recuperado de https://mangroves.elaw.org/es/node/70

GÓMEZ NIETO, Begoña y MARTÍNEZ DOMÍNGUEZ, Rocío, 2016. Los valores éticos en la responsabilidad social corporativa. Anagramas Rumbos y Sentidos de la Comunicación, 14(28).

HAYMAN, John, 1991. Investigación y educación ( $3^{\mathrm{a} e d .) . ~ B a r c e l o n a, ~(E s p a n ̃ a) . ~}$ Editorial: Ediciones Paidós.

HILL, Charles y JONES, Gareth, 2000. Administración estratégica un enfoque integrado. ( $8^{\mathrm{a}}$ ed). México: Editorial McGraw-Hill.

HERNÁNDEZ SAMPIERI, Roberto, FERNÁNDEZ COLLADO, Carlos y BAPTISTA LUCIO, María Pilar, 2004. Metodología de la investigación. México: Editorial McGraw-Hill.

INEGI, 2018. Sistema de Cuentas Nacionales de México. Cuentas Económicas y Ecológicas de México 2012. Preliminar. Año base 2008. México INEGI. Recuperado de https://www.inegi.org.mx/app/biblioteca/ficha.html?upc=702825 004151

KAPLAN, Robert y Norton, David, 1996. El cuadro de mando integral (The Balance Scorecard). Editorial: Gestión 2000. Madrid, (España).

KOTLER, Philip; BRADY, Mairead; GOODMAN, Malcom y HANSEN, Torben, 2009. Marketing Management. Harlow, UK. Editorial: Pearson Education Ltd.

MELLA VALENZUELA, Orlando, 2010. Grupos focales. Técnicas de investigación cuantitativa. Aspectos introductorios (p. 28). Santiago, Chile: CIDE.

PRIETO PIÑA, Damirya Danesa, 2015. Análisis de los factores del entorno bajo el enfoque de PESTEL y DAFO para el proyecto empresarial "FEQUIMA": Portal web de maquinarias, equipos y herramientas en Brasil. Sapienza Organizacional, 2(3),129-152.

PUBLÍMETRO, 2020. En Quintana Roo, cadenas hoteleras de EU y Europa mandan. Recuperado de https://www.publimetro.com.mx/mx/noticias/2015/10/19/ en-quintana-roo-cadenas-hoteleras-de-eu-y-europa-mandan.html

SANZ LEÓN, Alberto, 2015. RSC: La importancia de la gestión de los grupos de interés en las organizaciones. Recuperado de https://prevenblog.com/rsc-laimportancia-de-la-gestion-de-los-grupos-de-interes-en-las-organizaciones/ 
SECRETARÍA DE GOBERNACIÓN, 2015. ¿Sabías que los Programas Nacionales de Empresas y Derechos Humanos se relacionan con el desarrollo sostenible? Blog de la Secretaría de Gobernación del Gobierno de México. Recuperado de https://www.gob.mx/segob/articulos/sabias-que-los-programas -nacionales-de-empresas-y-derechos-humanos-se-relacionan-con-el-desarrollosostenible? idiom $=$ es

TAMAYO y TAMAYO, Mario, 2003. El proceso de la investigación Cinetífica (4a ed.). México. DF, (México). Editorial: MUSA S.A. de C.V.

VOLPENTESTA, Jorge Roberto, CHAÍN, Tomás., ALACAÍN, Marcelo Fabián, NIEVAS, Gustavo, SPINELLI, Héctor Eduardo, CORDERO, María Inés, CORTEJANA, Alicia y GRECO, Penélope, 2014. Identificación del impacto de la gestión de los stakeholders en las estructuras de las empresas que desarrollan estrategias de responsabilidad social empresarial. Universidad \& Empresa, 63-92. Recuperado de https://www.redalyc.org/articulo.oa?id=187232 713003

WACKERNAGEL, Mathis y REES, William, 2001. Nuestra huella Ecológica: Reduciendo el impacto humano sobre la tierra. Santiago de Chile, (Chile). Editorial: LOM Ediciones. 